Research Paper

\title{
Pulsed Radiofrequency Applied to the Sciatic Nerve Improves Neuropathic Pain by Down-regulating The Expression of Calcitonin Gene-related Peptide in the Dorsal Root Ganglion
}

\author{
Hao Ren ${ }^{1}$, Hailong Jin ${ }^{1}$, Zipu Jia ${ }^{1}$, Nan Ji2 ${ }^{\bowtie}$, Fang Luo ${ }^{1 凶}$ \\ 1. Department of Anesthesiology and Pain Management, Beijing Tiantan Hospital, Capital Medical University; \\ 2. Department of Neurosurgery, Beijing Tiantan Hospital, Capital Medical University. \\ $\triangle$ Corresponding authors: Fang Luo, M.D., Professor, Department of Anesthesiology and Pain Management, Beijing Tiantan Hospital, Capital Medical \\ University, Beijing 100050, P.R. China. Tel: +86-010- 67096664; Fax: +86-010-67050177. E-mail: luofangwt@yahoo.com and Nan Ji, M.D., Professor, Department of \\ Neurosurgery, Beijing Tiantan Hospital, Capital Medical University, Beijing 100050, P.R. China. Tel: +86-13910713896. E-mail: cnpsycho@163.com \\ (C) Ivyspring International Publisher. This is an open access article distributed under the terms of the Creative Commons Attribution (CC BY-NC) license \\ (https://creativecommons.org/licenses/by-nc/4.0/). See http://ivyspring.com/terms for full terms and conditions.
}

Received: 2017.04.10; Accepted: 2017.07.06; Published: 2018.01.01

\begin{abstract}
Background: Clinical studies have shown that applying pulsed radiofrequency (PRF) to the neural stem could relieve neuropathic pain (NP), albeit through an unclear analgesic mechanism. And animal experiments have indicated that calcitonin gene-related peptide (CGRP) expressed in the dorsal root ganglion (DRG) is involved in generating and maintaining NP. In this case, it is uncertain whether PRF plays an analgesic role by affecting CGRP expression in DRG.

Methods: Rats were randomly divided into four groups: Groups A, B, C, and D. In Groups C and D, the right sciatic nerve was ligated to establish the $\mathrm{CCl}$ model, while in Groups $\mathrm{A}$ and $\mathrm{B}$, the sciatic nerve was isolated without ligation. After 14 days, the right sciatic nerve in Groups $B$ and $D$ re-exposed and was treated with PRF on the ligation site. Thermal withdrawal latency (TWL) and hindpaw withdrawal threshold (HWT) were measured before PRF treatment (Day 0 ) as well as after 2, 4, 8, and 14 days of treatment. At the same time points of the behavioral tests, the right L4-L6 DRG was sampled and analyzed for CGRP expression using RT-qPCR and an enzyme-linked immunosorbent assay (ELISA).

Results: Fourteen days after sciatic nerve ligation, rats in Groups $C$ and $D$ had a shortened TWL $(P<0.001)$ and a reduced HWT $(P<0.001)$ compared to those in Groups A and B. After PRF treatment, the TWL of the rats in Group D gradually extended with HWT increasing progressively. Prior to PRF treatment (Day 0), CGRP mRNA expressions in the L4-L6 DRG of Groups $C$ and $D$ increased significantly $(P<0.001)$ and were 2.7 and 2.6 times that of Group A respectively. ELISA results showed that the CGRP content of Groups C and D significantly increased in comparison with that of Groups $A$ and $B(P<0.01)$. After PRF treatment, the mRNA expression in the DRG of Group D gradually decreased and the mRNA expression was 1.7 times that of Group $A$ on the 4th $\operatorname{day}(P>0.05)$. On the $8^{\text {th }}$ and $14^{\text {th }}$ days, the mRNA levels in Group $D$ were restored to those of Groups A and B. Meanwhile, the CGRP content of Group D gradually dropped over time, from $76.4 \mathrm{pg} / \mathrm{mg}$ (Day 0) to $57.5 \mathrm{pg} / \mathrm{mg}$ (Day 14).

Conclusions: In this study, we found that, after sciatic nerve ligation, rats exhibited apparent hyperalgesia and allodynia, and CGRP mRNA and CGRP contents in the L4-L6 DRG increased significantly. Through lowering CGRP expression in the DRG, PRF treatment might relieve the pain behaviors of NP.
\end{abstract}

Key words: Neuropathic pain, pulsed radiofrequency, analgesia, chronic constriction injury, dorsal root ganglion, calcitonin gene-related peptide

\section{Introduction}

Neuropathic pain (NP) has been recently redefined by the Neuropathic Pain Special Interest Group (NeuPSIG) as pain arising as a direct consequence of a lesion or disease affecting the somatosensory system [1]. Although there have been progresses in several studies on its mechanism and 
treatment, NP remains a type of pain which is clinically refractory. Pulsed radiofrequency (PRF) is a minimally invasive technique and differs from continuous radiofrequency (CRF) in several aspects. The radiofrequency current emitted in PRF has an interim period of $480 \mathrm{msec}$ following each $20 \mathrm{msec}$ emission, which allows heat to disperse to the surrounding tissues so that the temperature of the therapy target site will not exceed $42{ }^{\circ} \mathrm{C}$ through which it could avoid a series of side effects caused by irreversible nerve damage in the case of CRF [2]. Since its invention, PRF has been proven by an array of clinical studies in treating kinds of NP, including postherpetic neuralgia [3], painful diabetic neuropathy [4], trigeminal neuralgia [5, 6], etc.

Animal experiments have confirmed that PRF can perform actively in treating allodynia and hyperpathia in rat NP models [7-11], albeit through an unclear analgesic mechanism. Some researchers have speculated that PRF played an analgesic role via thermal effects. However, studies applying PRF to the dorsal root ganglion (DRG) or sciatic nerve have not demonstrated the irreversible effect of thermal damage $[12,13]$. In fact, more studies support the biological effects of PRF rather than its thermal effects. Calcitonin gene-related peptide (CGRP) is a neuropeptide consisting of 37 amino acid residues and it exists in humans and rats in two different CGRP subtypes: CGRPa and CGRP $\beta$ respectively. CGRPa is mainly produced in the central and peripheral nervous systems, especially in the DRG, trigeminal ganglion and so on. The primary sensory fibers in the DRG project to laminae I and II of the spinal dorsal horn, the majority of which are pain-conducting $A \delta$ and $C$ fibers $[14,15]$. At the spinal cord level, CGRP plays an important role in chronic pain through facilitating the introduction of synaptic pain information via both protein-kinase-A along with protein-kinase- $C$ second-messenger pathways [16-18] and participating in the generation and maintenance of allodynia as well as hyperpathia [19, 20].

Studies have revealed that applying PRF to the peripheral nerve of normal rats can reduce the proportion of CGRP-positive neurons in the DRG [21], which suggests that PRF may control the pain symptoms of NP by affecting CGRP expression in the pain transduction pathway. In this study, we employed a rat chronic constriction injury (CCI) model to simulate NP. And we applied PRF to the oppressed portion of the rat sciatic nerve to investigate the pain behaviors at multiple time points before and after PRF treatment and to examine CGRP mRNA and CGRP levels in the L4-L6 DRG, thereby elucidating the possible mechanism of analgesic effect of PRF.

\section{Methods}

All procedures on animals were approved by the Beijing Neurosurgical Institute Experimental Animal Welfare Ethics Committee. The 4-month-old adult male Sprague-Dawley rats (220-250 g) used in this experiment were provided by Vital River Laboratories, Beijing and were raised in a 12-hour light-dark alternation environment at $22-24{ }^{\circ} \mathrm{C}$. One hundred and twenty rats were randomly divided into four groups and were treated as follows:

Group A $(n=30)$ : sham-CCI and sham-PRF, in which the right sciatic nerve was exposed, without nerve ligation. Fourteen days after the surgery, the right sciatic nerve was exposed again. A trocar with an electrode needle used for PRF treatment was placed at the sciatic nerve, without applying a pulse RF current.

Group B ( $n=30)$ : sham-CCI and PRF, in which the right sciatic nerve was exposed, without nerve ligation. Fourteen days after the surgery, the right sciatic nerve was re-exposed and treated with PRF.

Group C $(n=30)$ : CCI procedure and sham-PRF, in which the right sciatic nerve was exposed and ligated to create the CCI model. Fourteen days after the surgery, the right sciatic nerve was once again exposed. A trocar with an electrode needle used for PRF treatment was placed at the sciatic nerve, without applying a pulse RF current.

Group D $(n=30)$ : CCI procedure and PRF procedure, in which the right sciatic nerve was exposed and ligated to create the CCI model. Fourteen days after the surgery, with the exposure of the right sciatic nerve, PRF treatment was conducted.

Fourteen days after the sciatic nerve ligation surgery, each group was subjected to pain behavioral tests before (Day 0) and after 2, 4, 8, and 14 days of PRF treatment. Following the same time points of the behavioral tests, the rats were sacrificed, and the right L4-L6 DRG was sampled to analyze the CGRP mRNA expression and neuropeptide content.

\section{Sciatic nerve $\mathbf{C C l}$ model}

The CCI model was established on the basis of the method from Bennett and Xie [22]. After being anesthetized via intraperitoneal injection of sodium pentobarbital $(40 \mathrm{mg} / \mathrm{kg})$, the rat's sciatic nerve was exposed, and then ligated in four strips with 1-mm spacing using $4 / 0$ chromium catgut, and just not to block the surface vessel of the sciatic nerve is the most appropriate. The wound was closed in layers and cleaned. 


\section{PRF}

Fourteen days after the surgery, PRF treatment was performed. The rat's right sciatic nerve was once again exposed, and the trocar (PMF-21-50-2, Baylis, Canada) and electrode needle (PMK-21-50, Baylis, Canada) for PRF treatment were placed vertically at where the sciatic nerve was ligated and after that connected to a PRF generator (PMG-230, Baylis Medical, Inc., Montreal, Canada). The settings were a pulse emission frequency of $2 \mathrm{~Hz}$, a voltage of $45 \mathrm{~V}$, a treatment period of 120 seconds and with the temperature less than $42{ }^{\circ} \mathrm{C}$. While rats under the sham-PRF treatment took a trocar with electrode needle but no PRF current.

\section{Behavioral tests}

Rats in each group $(n=6)$ were subjected to pain behavioral tests before PRF treatment (Day 0) and after 2, 4, 8, and 14 days of treatment respectively. Thermal withdrawal latency (TWL) and hindpaw withdrawal threshold (HWT) were used to measure the thermal pain threshold and mechanical pain threshold of the rat's right hind paw.

\section{TWL}

In accordance with the methodology of Hargreaves et al [23], the rats were placed in a bottomless cage made of a plexiglass plate. The mobile radiant heat source of the Plantar Test Instrument (Ugo Basile 37370) which was located under a quartz glass plate would be aligned to the surface of the third metatarsal bone of rats' hind paw. The instrument automatically recorded the duration from the start of radiation to the emergence of the escape reflex, i.e., TWL (sec). The measurements were repeated thrice in 10-min intervals at the same spot, and later averaged. The maximum heat exposure time was set within 22.5 seconds, to avoid burns to the rat's plantar surface.

\section{HWT}

In line with the research of Vivancos et al [24], absolute withdrawal thresholds were measured by an electronic von Frey apparatus (Electronic von Frey Anesthesiometer 2390, IITC, Inc.). The rat was placed in an experimental cage with a perforated metal sheet (mesh size $0.5 * 0.5 \mathrm{~cm}$ ) and the measurement commenced after 10-15 minutes. The non-footpad area of the rat's middle plantar was stimulated by an electronic von Frey rigid tip through the mesh bottom, and the stimulated spots were the same as those in the case of TWL. The operator gradually increased the pressure to induce the foot withdrawal response in the rat, in which the maximum strength $(\mathrm{g})$ was recorded by the instrument. The rat would be placed on the cage for $10-15$ minutes before commencing the measurement.

\section{CGRP expression}

Before PRF treatment (Day 0) and on the second, fourth, eighth and fourteenth day after the treatment, the right L4-L6 DRG of the rats ( $n=6 /$ group/time point) was quickly excised after anesthesia and rinsed with saline to remove excess tissue and blood. All operations were conducted at $0-4{ }^{\circ} \mathrm{C}$, and the sampled tissue specimens were stored immediately in liquid nitrogen for later RT-qPCR or enzyme-linked immunosorbent assay (ELISA) analysis.

\section{RT-qPCR}

The sample was homogenized, from which total RNA was extracted using the Trizol reagent (Invitrogen, Carlsbad, CA) and quantified through light absorption at $260 \mathrm{~nm}$. The first strand cDNA was obtained through reverse transcription using the ProtoScript TM First Strand cDNA Synthesis Kit (NEB, USA) according to the manufacturer's instruction. The CGRP primer sequences are listed in Table 1, with $\beta$-actin as the reference gene. CGRP mRNA expression levels in the sample tissues were detected with the cDNA as the template using the fluorescent quantitative PCR method and the Fast SYBR Green Master Mix qPCR kit (Thermo Fisher Scientific, USA) on the ABI StepOnePlus TM System (ABI, USA). The reaction conditions referred to the user's manuals of the StepOnePlus TM System and the Fast SYBR Green Master Mix qPCR kit. Each sample was made into three aliquots and subjected to RT-qPCR with averaged the result made. The resultant quantification cycle $(\mathrm{Cq})$ was adopted to calculate the relative amount of CGRP mRNA expression by aid of the $2^{-}-\Delta \triangle \mathrm{CT}$ method [25] and $\beta$-actin as the reference.

Table 1. Primer sequences for the rat genes characterized in this experiment

\begin{tabular}{llll}
\hline Gene & $\begin{array}{l}\text { GenBank } \\
\text { numbers }\end{array}$ & $\begin{array}{l}\text { Product } \\
\text { length } \\
(\mathrm{bp})\end{array}$ & \\
\hline CGRPa & NM_017338 160 & $\begin{array}{l}\text { Forward CAGGAGGAGGAACAGGAGGCT } \\
\text { Reverse TCTTGCCAGGTGCTCCAACC }\end{array}$ \\
$\beta$-Actin NM_031144 150 & $\begin{array}{l}\text { Forward CCCATCTATGAGGGTTACG } \\
\text { Reverse }\end{array}$ \\
\hline
\end{tabular}

\section{ELISA quantification of CGRP}

The specimens were collected as described above, after which accurately weighed and homogenized. The homogenized sample was centrifuged with the supernatant collected, from which the CGRP content in the L4-L6 DRG was 
analyzed. The experiment stuck to the manufacturer's instructions for the ELISA kit (Elabscience Biotechnology Co, Ltd., Wuhan, China). Absorbance at $450 \mathrm{~nm}$ was measured through a microplate reader, and a standard curve was generated. The CGRP content of the sample was calculated based on this.

\section{Statistical Analysis}

All the data were presented as the mean \pm SEM and analyzed with SPSS 20.0. For behavioral test data, two-way repeated measures ANOVA was used, and the Bonferroni post-test for inter-group comparisons. RT-qPCR results of each time point were analyzed in single-factor ANOVA, and the SNK method was utilized for pair-wise inter-group comparisons. ELISA results were analyzed using two-factor ANOVA, whereas the Bonferroni post-test was in use for

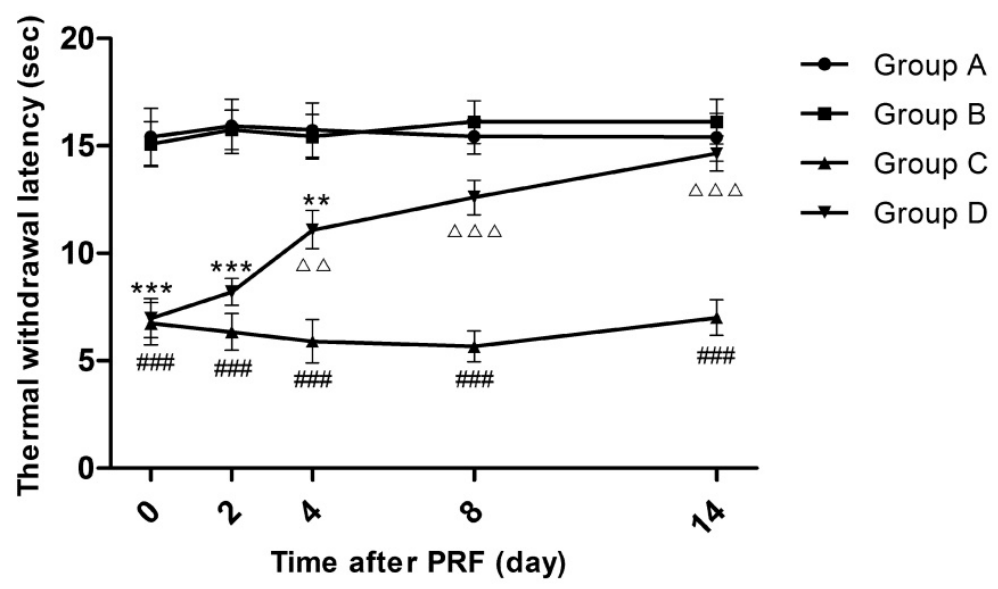

Figure 1. Effect of pulsed radiofrequency (PRF) on the thermal withdrawal latency after sciatic nerve ligation *: Comparison between Group $D$ and Group $A(* * *: P<0.001$; **: P<0.01); \#: Comparison between Group $C$ and Group A (\#\#: P<0.001); $\Delta$ : Comparison between Group $C$ and Group $D(\Delta \Delta \Delta: P<0.001 ; \Delta \Delta: P<0.01)$. Data are expressed as the mean \pm SEM.

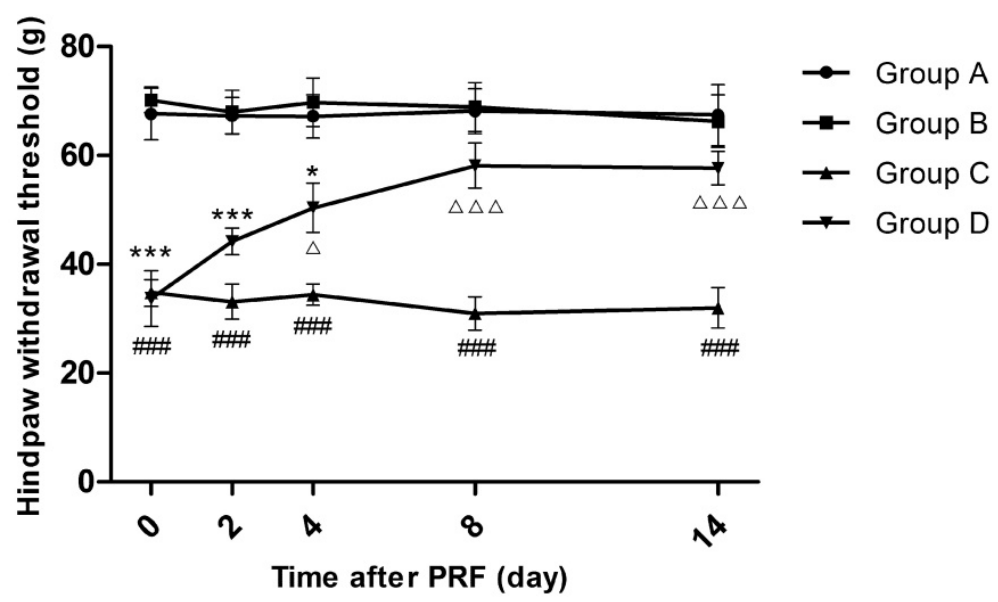

Figure 2. Effect of pulsed radiofrequency (PRF) on the hindpaw withdrawal threshold after sciatic nerve ligation *: Comparison between Group $D$ and Group $A(* * *:$ $\mathrm{P}<0.001$; *: $\mathrm{P}<0.05$ ); \#: Comparison between Group $\mathrm{C}$ and Group A (\#\#: $\mathrm{P}<0.001$ ); $\Delta$ : Comparison between Group $C$ and Group $D(\Delta \Delta \Delta: P<0.001 ; \Delta: P<0.05)$. Data are expressed as the mean $\pm S E M$. inter-group comparisons. $\mathrm{P}<0.05$ was set as the significance level of the tests.

\section{Results}

\section{Behavioral tests}

TWL

Fourteen days after sciatic nerve ligation, while before PRF treatment (Day 0), the TWLs of Groups C and $\mathrm{D}$ were significantly shortened compared to those of Groups A and B $(P<0.001)$, whereas there was no significant TWL difference between Groups A and B and so was the situation between Groups C and D. Four days after PRF treatment, the average TWL of Group D rose from 7.0 to 11.1 seconds, being significantly extended compared to that of Group C $(\mathrm{P}<0.01)$. On the $8^{\text {th }}$ and $14^{\text {th }}$ days after PRF treatment, the average TWLs of Group D returned to 12.6 seconds and 14.7 seconds respectively, being insignificantly different from those of Groups A and B but significantly longer than the average TWLs of Group C $(\mathrm{P}<0.001)$. At each time point, the average TWLs of Group $C$ were always distinctly lower than those of Group A or Group B (P<0.001). (Figure 1)

HWT

The trend of how HWT changed in each group was similar to that of the TWL in each group. Before PRF treatment, the average HWTs of Groups C and D were overtly lower than those of Groups A and B $(\mathrm{P}<0.001)$, whereas the average HWT differences between Groups A and B as well as between Groups C and D were marginal. On the $4^{\text {th }}$ day after PRF treatment, rats in Group D recovered to $50.4 \mathrm{~g}$, which was apparently higher than that in Group C $(\mathrm{P}<0.05)$ but still not reaching the levels in Group A $(\mathrm{P}<0.05)$. On the $8^{\text {th }}$ day and $14^{\text {th }}$ day after PRF treatment, the HWTs of Group $D$ further recovered but there was no significant difference from those of Groups A and B. (Figure 2)

\section{RT-qPCR}

Fourteen days after sciatic nerve ligation and before PRF treatment (Day 0), the CGRP mRNA expression in the DRG of Groups C and D significantly increased $(P<0.001)$ and was 2.7 times and 2.6 times respectively while the level of CGRP mRNA expression in the DRG of Group A. there were no differences between Groups $C$ and D. After PRF treatment, the mRNA 
expression in the DRG of Group D decreased gradually, and on the $4^{\text {th }}$ day, it was 1.7 times that of Group A, albeit an insignificant difference ( $P>0.05)$. On the $8^{\text {th }}$ and $14^{\text {th }}$ days, the mRNA levels in Group D were restored to levels of Groups A and B. (Figure 3)

\section{ELISA}

On Day 0, the CGRP contents in the L4-L6 DRG of Groups A and B were significantly increased compared with those in Groups C and D $(\mathrm{P}<0.01)$. After PRF treatment, the CGRP content of Group D decreased from $76.4 \mathrm{pg} / \mathrm{mg}$ (Day 0) to $57.5 \mathrm{pg} / \mathrm{mg}$ (Day 14). On Day 4, although the CGRP content of Group D was still higher than that of Group A or Group B, the difference was not obvious. On Day 8, the CGRP content of Group D went up again to the level of Group A and B. The CGRP content of Group C was consistently higher than that of Group A or Group B $(\mathrm{P}<0.001)$. (Figure 4)

\section{Discussion}

\section{PRF performance on the oppressed site of the sciatic nerve improved hyperalgesia and allodynia in the rat $\mathrm{CCl}$ model.}

In this study, the TWLs of rats in Groups C and $D$ were shortened and the HWTs were declined on the 14th day after sciatic nerve ligation indicating the emergence of allodynia and hyperpathia, which proved the successful establishment of the CCI model. The CCI model is a widely used, reliable NP model which can simulate clinical symptoms of human NP such as spontaneous pain, allodynia, and
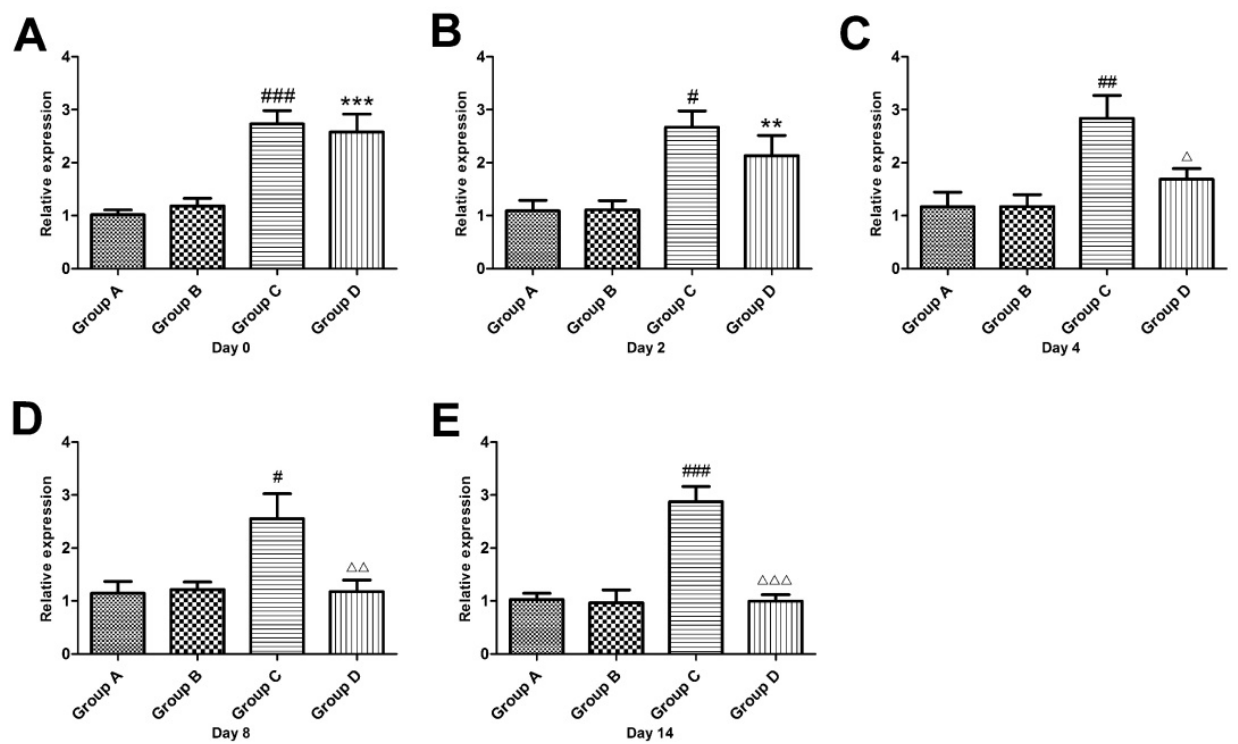

Figure 3. Effect of pulsed radiofrequency treatment on the calcitonin gene-related peptide mRNA levels in the dorsal root ganglion after sciatic nerve ligation *: Comparison between Group D and Group A (***: $\mathrm{P}<0.001$; **: $\mathrm{P}<0.01$ ); \#: Comparison between Group $\mathrm{C}$ and Group A (\#\#: $\mathrm{P}<0.001$; \#\#: $\mathrm{P}<0.01$; \#: $\mathrm{P}<0.05$ ); $\Delta$ : Comparison between Group $C$ and Group $D(\Delta \Delta \Delta: P<0.001 ; \Delta \Delta: P<0.01 ; \Delta: P<0.05)$. Data are expressed as the mean \pm SEM. hyperpathia, among others. On the $2^{\text {nd }}$ day after the application of PRF to the oppressed site of the sciatic nerve stem (Group D), thermal hyperalgesia and mechanical allodynia were only slightly relieved. On the $4^{\text {th }}$ day, approximately $50 \%$ of pain relief was achieved. The pain was mostly relieved on the $8^{\text {th }}$ day and it was almost completely relieved on the $14^{\text {th }}$ day. These results are consistent with those in previous studies [7, 26, 27] and confirm that PRF on an oppressed site of a peripheral nerve can gradually and significantly alleviate the hyperalgesia and allodynia of an NP model.

Consisting with the time for PRF to take effect on analgesia, i.e. approximately 4 days after PRF treatment of a peripheral nerve in the spared nerve injury (SNI) model reported by Vallejo et al. [27], it also took four days for PRF treatment of the CCI model to achieve satisfactory effectiveness. Erdine et al. [28] believed that PRF mainly acts on the A $\delta$ and C fibers of the rat's primary afferent nerve fibers and achieves its analgesic effect by interfering with the integrity of incoming nerve impulses. Tun et al. [13] considered that PRF might interfere or block the signal transduction of nerve pathways by causing the separation of myelin in nerve axons and might further cause reversible inhibition of nerve cell synapses. However, our investigation, consistent with most studies, did not show the immediate interfering effect of PRF on the transduction of nerve impulses, suggesting that PRF may function through other analgesic mechanisms.

In this study, PRF acted directly on the sciatic nerve stem of the NP model. Currently, clinical PRF targets of NP include peripheral nerve and the DRG, and in both cases, the treatment exhibited satisfactory effectiveness. PRF treatment on peripheral nerve only requires simple operations, has little puncture risk, and can be positioned using ultrasonography instead of radiological imaging equipment, such as X-rays, $\mathrm{CT}$, etc., thus having certain advantages. However, when PRF acts on the peripheral nerve or DRG of NP, which approach improves better efficacy is still in dispute. Further in-depth studies will lay the foundation for the 
clinical practice of PRF treatment on NP.

\section{CGRP expression increased in the rat $\mathrm{CCI}$ model.}

In this study, it was found that 14 days after sciatic nerve ligation and after the successful establishment of the rat model (Groups C and D), CGRP mRNA expression in the L4-L6 DRG significantly increased, and the CGRP content was significantly higher than that of Group A or Group B (which had no sciatic nerve ligation). This result is different from that gained by Bennett et al. [29], who found that the number of small-sized neurons expressing CGRP in the L4 and L5 DRG of the rat CCI model continuously decreased for 2-3 months, and on the $10^{\text {th }}$ and $20^{\text {th }}$ days after the establishment of the model, the CGRP content of the nerve injury region of the corresponding spinal dorsal horn decreased by $16 \%$ and $19 \%$ accordingly. Currently, findings on CGRP changes in the nociception transduction pathway of NP model have been inconsistent. The majority of the studies report that the animal NP models derived from peripheral nerve injury exhibit up-regulated CGRP expression in the DRG [30-32] or spinal cord $[32,33]$ and the accumulation of CGRP at the nerve injury site is due to blocked CGRP transport[34, 35]. After peripheral nerve injury, many medium and large DRG neurons begin to express CGRP and play an important role in generating and maintaining pain behaviors [36, 37]. Actions antagonistic to CGRP can ease the pain behaviors [19, 38]. Our findings support there is an increase of CGRP expression in NP rats. The cause of the inconsistent findings in previous studies may be associated with the CGRP detection method (measuring the number of immunologically positive cells or the optical

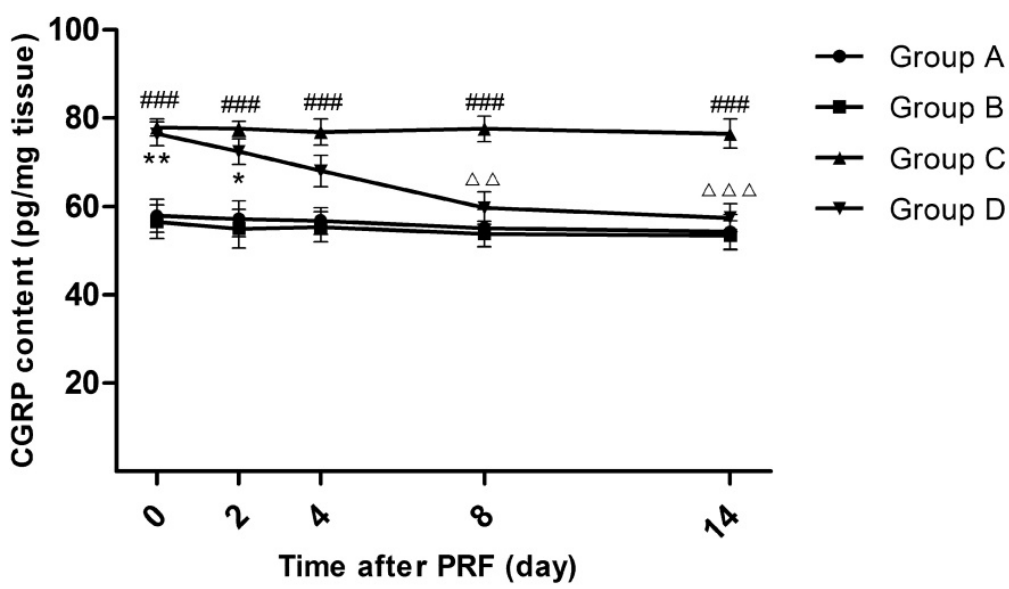

Figure 4. Effect of pulsed radiofrequency (PRF) treatment on the calcitonin gene-related peptide content in the dorsal root ganglion after sciatic nerve ligation *: Comparison between Group D and Group A (**: P<0.01; *: P<0.05); \#: Comparison between Group C and Group A (\#\#: $P<0.001) ; \Delta$ : Comparison between Group $C$ and Group $D(\Delta \Delta \Delta: P<0.001 ; \Delta \Delta: P<0.01)$. Data are expressed as the mean \pm SEM. density of positive reaction, etc.) or the applications of different peripheral nerve injury models (SNI model, CCI model, sciatic axotomy, etc.).

\section{PRF might play an analgesic role through reducing CGRP expression in the DRG.}

The RT-qPCR results showed that the relative amount of CGRP mRNA expression in the DRG of Group D began to decline on the $2^{\text {nd }}$ day after PRF treatment. And on the $4^{\text {th }}$ day, it was restored to a normal level (showing no difference from Group A). The ELISA results revealed that after PRF treatment, the CGRP content in the DRG of Group D was decreased and then restored to the same level of Group A on the $8^{\text {th }}$ day. The above-mentioned results indicated that PRF could inhibit the transcription and translation of CGRP in the rat's DRG. At present, no consensus on the analgesic mechanism of PRF has been reached, and it is believed that the mechanism might be related to the influences from a variety of neuropeptides, proteins, and inflammatory factors $[26,27,39]$, which may not be independent of each other. Moreover, what type of connection and which is the core part of the PRF role remain unknown. CGRP is mainly synthesized in the DRG, in which primary sensory neurons project nerve fibers to laminae I and II of the spinal dorsal horn [40]. Once peripheral nerve injury occurs, primary sensory fibers that are projected to the spinal dorsal horn in the DRG release CGRP, $\mathrm{P}$ substances, etc., leading to the activation of glial cells, which in turn release various pain regulators such as tumor necrosis factor- $\alpha$ (TNF-a), interleukin-6 (IL-6), and nerve growth factor etc., which are involved in central sensitization [41-43]. It was hypothesized that PRF treatment disrupts the above-described chain reaction through inhibiting CGRP expression in the DRG, which might be one of the analgesic mechanisms of the treatment. However, what type of role the CGRP mechanism plays in easing NP after PRF treatment and its relationship with other mechanisms still requires further investigation.

\section{Limitations}

Although our study showed that PRF can down-regulate CGRP expression in the DRG of the rat CCI model and reduce pain behaviors, the detailed relationship among PRF, CGRP and pain behaviors still requires further experimental clarifications. For instance, after applying a CGRP antagonist or supplementing CGRP, 
the role of PRF is monitored. This study examined only the translation and transcription level of CGRP in the DRG. The CGRP expression in other parts of the nociception pathway, such as the dorsal horn of the spinal cord, sciatic nerve, etc., was not investigated. After sciatic nerve ligation, anterograde transport of CGRP to nerve endings from the DRG is blocked, and CGRP accumulated at the ligation site; with the recanalization of nerve on the ligation site, the CGRP accumulation is relieved $[35,37]$. Whether PRF directly affects the axial transport of CGRP in peripheral nerve is uncertain, and our experiments did not reveal through which mechanism PRF affects CGRP expression and which physical characteristics of PRF (e.g., the current versus the electrical field) generate the biological effect. The therapeutic effect of PRF may be derived from multiple mechanisms that may intercrossed with each other. Our study did not reveal the connection between the CGRP mechanism and the other PRF analgesic mechanisms reported previously. We observed changes in pain behaviors and CGRP expressions only 14 days after PRF treatment. However, longer follow-up is necessary to ascertain whether PRF has long-term efficacy.

\section{Conclusions}

In this study, we found that after sciatic nerve ligation, rats exhibited apparent hyperalgesia and allodynia and that the CGRP mRNA and CGRP content in the L4-L6 DRG significantly increased. All in all, the research revealed that PRF treatment might relieve NP pain behavioral performances by lowering CGRP expression in the DRG.

\section{Abbreviations}

neuropathic pain: NP; calcitonin gene-related peptide: CGRP; dorsal root ganglion: DRG; Thermal withdrawal latency: TWL; hindpaw withdrawal threshold: HWT; enzyme-linked immunosorbent assay: ELISA; Pulsed radiofrequency: PRF; continuous radiofrequency: CRF; chronic constriction injury model: $\mathrm{CCI}$; spared nerve injury: SNI; tumor necrosis factor-a: TNF-a; interleukin-6: IL-6

\section{Acknowledgements}

This study was supported by Foundation for The Excellent Medical Staff of Beijing (No. 2011-3-034 and No. 2014-3-035). Ren Hao, Jin Hailong, and Jia Zipu contributed equally to this work. Luo Fang and Ji Nan corresponded equally to this work in designing and supervising the project.

\section{Competing Interests}

The authors have declared that no competing interest exists.

\section{References}

1. Treede RD, Jensen TS, Campbell JN, Cruccu G, Dostrovsky JO, Griffin JW, et al. Neuropathic pain: redefinition and a grading system for clinical and research purposes. Neurology. 2008; 70: 1630-5.

2. Sluijter ME, Cosman ER, Rittmann WB, Van Kleef M. The effects of pulsed radiofrequency fields applied to the dorsal root ganglion - a preliminary report. Pain Clinic. 1998; 11: 109-17.

3. Ke M, Yinghui F, Yi J, Xeuhua H, Xiaoming L, Zhijun C, et al. Efficacy of pulsed radiofrequency in the treatment of thoracic postherpetic neuralgia from the angulus costae: a randomized, double-blinded, controlled trial. Pain Physician. 2013; 16: 15-25.

4. Naderi Nabi B, Sedighinejad A, Haghighi M, Biazar G, Hashemi M, Haddadi $\mathrm{S}$, et al. Comparison of transcutaneous electrical nerve stimulation and pulsed radiofrequency sympathectomy for treating painful diabetic neuropathy. Anesth Pain Med. 2015; 5: e29280.

5. Fang L, Ying S, Tao W, Lan M, Xiaotong Y, Nan J. 3D CT-guided pulsed radiofrequency treatment for trigeminal neuralgia. Pain Pract. 2014; 14: 16-21.

6. Fang L, Tao W, Jingjing L, Nan J. Comparison of High-voltage- with Standard-voltage Pulsed Radiofrequency of Gasserian Ganglion in the Treatment of Idiopathic Trigeminal Neuralgia. Pain Pract. 2015; 15: 595-603.

7. Li DY, Meng L, Ji N, Luo F. Effect of pulsed radiofrequency on rat sciatic nerve chronic constriction injury: a preliminary study. Chin Med J (Engl). 2015; 128: 540-4

8. Perret DM, Kim DS, Li KW, Sinavsky K, Newcomb RL, Miller JM, et al. Application of pulsed radiofrequency currents to rat dorsal root ganglia modulates nerve injury-induced tactile allodynia. Anesth Analg. 2011; 113: 610-6

9. Huang $\mathrm{YH}$, Hou SY, Cheng JK, $\mathrm{Wu} \mathrm{CH}$, Lin CR. Pulsed radiofrequency attenuates diabetic neuropathic pain and suppresses formalin-evoked spinal glutamate release in rats. Int J Med Sci. 2016; 13: 984-91.

10. Liu CK, Liao WT, Chu YC, Yang $\mathrm{CH}$, Chen $\mathrm{KH}, \mathrm{Wu} \mathrm{CH}$, et al. Pulsed Radiofrequency Attenuates Complete Freund's Adjuvant-Induced Epigenetic Suppression of Potassium Chloride Cotransporter 2 Expression. Pain Med. 2016

11. Yeh CC, Wu ZF, Chen JC, Wong CS, Huang CJ, Wang JS, et al. Association between extracellular signal-regulated kinase expression and the anti-allodynic effect in rats with spared nerve injury by applying immediate pulsed radiofrequency. BMC Anesthesiol. 2015; 15: 92.

12. Podhajsky RJ, Sekiguchi Y, Kikuchi S, Myers RR. The histologic effects of pulsed and continuous radiofrequency lesions at 42 degrees $C$ to rat dorsal root ganglion and sciatic nerve. Spine (Phila Pa 1976). 2005; 30: 1008-13.

13. Tun K, Cemil B, Gurcay AG, Kaptanoglu E, Sargon MF, Tekdemir I, et al. Ultrastructural evaluation of pulsed radiofrequency and conventional radiofrequency lesions in rat sciatic nerve. Surg Neurol. 2009; 72: 496-500; discussion 501.

14. McCarthy PW, Lawson SN. Cell type and conduction velocity of rat primary sensory neurons with calcitonin gene-related peptide-like immunoreactivity. Neuroscience. 1990; 34: 623-32.

15. Hokfelt T. Neuropeptides in perspective: the last ten years. Neuron. 1991; 7: 867-79.

16. Ryu PD, Gerber G, Murase K, Randic M. Actions of calcitonin gene-related peptide on rat spinal dorsal horn neurons. Brain Res. 1988; 441: 357-61.

17. Miletic $\mathrm{V}$, Tan $\mathrm{H}$. Iontophoretic application of calcitonin gene-related peptide produces a slow and prolonged excitation of neurons in the cat lumbar dorsal horn. Brain Res. 1988; 446: 169-72.

18. Pezet S, McMahon SB. Neurotrophins: mediators and modulators of pain. Annu Rev Neurosci. 2006; 29: 507-38.

19. Lee SE, Kim JH. Involvement of substance $\mathrm{P}$ and calcitonin gene-related peptide in development and maintenance of neuropathic pain from spinal nerve injury model of rat. Neurosci Res. 2007; 58: 245-9.

20. Sun RQ, Lawand NB, Willis WD. The role of calcitonin gene-related peptide (CGRP) in the generation and maintenance of mechanical allodynia and hyperalgesia in rats after intradermal injection of capsaicin. Pain. 2003; 104: 201-8.

21. Hamann W, Abou-Sherif S, Thompson S, Hall S. Pulsed radiofrequency applied to dorsal root ganglia causes a selective increase in ATF3 in small neurons. Eur J Pain. 2006; 10: 171-6.

22. Bennett GJ, Xie YK. A peripheral mononeuropathy in rat that produces disorders of pain sensation like those seen in man. Pain. 1988; 33: 87-107.

23. Hargreaves K, Dubner R, Brown F, Flores C, Joris J. A new and sensitive method for measuring thermal nociception in cutaneous hyperalgesia. Pain. 1988; 32: 77-88.

24. Vivancos GG, Verri WA, Jr., Cunha TM, Schivo IR, Parada CA, Cunha FQ, et al. An electronic pressure-meter nociception paw test for rats. Braz J Med Biol Res. 2004; 37: 391-9.

25. Livak KJ, Schmittgen TD. Analysis of relative gene expression data using real-time quantitative PCR and the 2(-Delta Delta $\mathrm{C}(\mathrm{T})$ ) Method. Methods. 2001; 25: 402-8.

26. Lee JB, Byun JH, Choi IS, Kim Y, Lee JS. The effect of pulsed radiofrequency applied to the peripheral nerve in chronic constriction injury rat model. Ann Rehabil Med. 2015; 39: 667-75.

27. Vallejo R, Tilley DM, Williams J, Labak S, Aliaga L, Benyamin RM. Pulsed radiofrequency modulates pain regulatory gene expression along the nociceptive pathway. Pain Physician. 2013; 16: E601-13. 
28. Erdine S, Bilir A, Cosman ER, Cosman ER. Ultrastructural changes in axons following exposure to pulsed radiofrequency fields. Pain Pract. 2009; 9: 407-17.

29. Bennett GJ, Kajander KC, Sahara Y, Iadarola MJ, Sugimoto T. Neurochemical and anatomical changes in the dorsal horn of rats with an experimental painful peripheral neuropathy. In: Cervero F, Bennett GJ, Headley PM, ed. Processing of Sensory Information in the Superficial Dorsal Horn of the Spinal Cord. Boston: Springer; 1989:463-71.

30. Hirose K, Iwakura N, Orita S, Yamashita M, Inoue G, Yamauchi K, et al. Evaluation of behavior and neuropeptide markers of pain in a simple, sciatic nerve-pinch pain model in rats. Eur Spine J. 2010; 19: 1746-52.

31. Murakami K, Kuniyoshi K, Iwakura N, Matsuura Y, Suzuki T, Takahashi K, et al. Vein wrapping for chronic nerve constriction injury in a rat model: study showing increases in VEGF and HGF production and prevention of pain-associated behaviors and nerve damage. J Bone Joint Surg Am. 2014; 96: 859-67.

32. Miki K, Fukuoka T, Tokunaga A, Noguchi K. Calcitonin gene-related peptide increase in the rat spinal dorsal horn and dorsal column nucleus following peripheral nerve injury: up-regulation in a subpopulation of primary afferent sensory neurons. Neuroscience. 1998; 82: 1243-52.

33. Ma W, Bisby MA. Ultrastructural localization of increased neuropeptide immunoreactivity in the axons and cells of the gracile nucleus following chronic constriction injury of the sciatic nerve. Neuroscience. 1999; 93: 335-48.

34. Schafers M, Geis C, Brors D, Yaksh TL, Sommer C. Anterograde transport of tumor necrosis factor-alpha in the intact and injured rat sciatic nerve. J Neurosci. 2002; 22: 536-45.

35. Zheng LF, Wang R, Xu YZ, Yi XN, Zhang JW, Zeng ZC. Calcitonin gene-related peptide dynamics in rat dorsal root ganglia and spinal cord following different sciatic nerve injuries. Brain Res. 2008; 1187: 20-32.

36. Hu P, Bembrick AL, Keay KA, McLachlan EM. Immune cell involvement in dorsal root ganglia and spinal cord after chronic constriction or transection of the rat sciatic nerve. Brain Behav Immun. 2007; 21: 599-616.

37. Ishikawa T, Miyagi M, Yamashita M, Kamoda H, Eguchi Y, Arai G, et al. In-vivo transfection of the proopiomelanocortin gene, precursor of endogenous endorphin, by use of radial shock waves alleviates neuropathic pain. J Orthop Sci. 2013; 18: 636-45.

38. La Rana G, Russo R, D'Agostino G, Sasso O, Raso GM, Iacono A, et al. AM404, an anandamide transport inhibitor, reduces plasma extravasation in a model of neuropathic pain in rat: role for cannabinoid receptors. Neuropharmacology. 2008; 54: 521-9.

39. Park HW, Ahn SH, Son JY, Kim SJ, Hwang SJ, Cho YW, et al. Pulsed radiofrequency application reduced mechanical hypersensitivity and microglial expression in neuropathic pain model. Pain Med. 2012; 13: 1227-34.

40. Rethelyi M, Metz CB, Lund PK. Distribution of neurons expressing calcitonin gene-related peptide mRNAs in the brain stem, spinal cord and dorsal root ganglia of rat and guinea-pig. Neuroscience. 1989; 29: 225-39.

41. McMahon SB, Cafferty WB, Marchand F. Immune and glial cell factors as pain mediators and modulators. Exp Neurol. 2005; 192: 444-62.

42. Marchand F, Perretti M, McMahon SB. Role of the immune system in chronic pain. Nat Rev Neurosci. 2005; 6: 521-32.

43. Wu FX, Bian JJ, Miao XR, Huang SD, Xu XW, Gong DJ, et al. Intrathecal siRNA against Toll-like receptor 4 reduces nociception in a rat model of neuropathic pain. Int J Med Sci. 2010; 7: 251-9. 